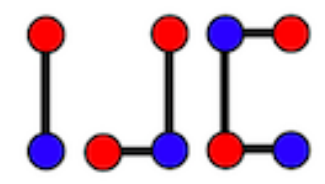

\title{
Some methods for constructing some classes of graceful uniform trees
}

\author{
I Nengah Suparta ${ }^{a}$, I Dewa Made Agus Ariawan ${ }^{a}$ \\ ${ }^{a}$ Department of Mathematics, Universitas Pendidikan Ganesha, Singaraja, Indonesia \\ nengah.suparta@undiksha.ac.id, iddeagus@gmail.com
}

\begin{abstract}
A tree $T(V, E)$ is graceful if there exists an injective function $f$ from the vertex set $V(T)$ into the set $\{0,1,2, \ldots,|V|-1\}$ which induces a bijective function $f^{\prime}$ from the edge set $E(T)$ onto the set $\{1,2, \ldots,|E|\}$, with $f^{\prime}(u v)=|f(u)-f(v)|$ for every edge $\{u, v\} \in E$. Motivated by the conjecture of Alexander Rosa (see [1]) saying that all trees are graceful, a lot of works have addressed gracefulness of some trees. In this paper we show that some uniform trees are graceful. This results will extend the list of graceful trees.
\end{abstract}

Keywords: Caterpillar, uniform caterpillar, uniform super lobster, uniform distant tree, graceful

Mathematics Subject Classification : 05C12, $05 \mathrm{C} 78$

DOI: 10.19184/ijc.2018.2.2.7

\section{Introduction}

Let $G(V, E)$ be a graph of $|V(G)|$ vertices and $|E(G)|$ edges. Graphs labelling is a notorious topic in graph theory. This is not because of its interesting combinatorial property of graphs labelling but also because of applications side such as X-ray crystallography, coding theory, circuit design, or astronomy (see [5]).

In year 1967, Alexander Rosa introduced $\beta$ labelling or $\beta$-valuations which was popularized by Solomon W. Golomb as graceful labelling. A graceful labelling for the graph $G(V, E)$ is an injective function $f$ from the vertex set $V(G)$ into the set $\{0,1,2, \ldots,|E(G)|\}$ which induces edge labels $|f(u)-f(v)|$ for all $\{u, v\} \in E(G)$ which are all distinct. A graph which admits a graceful

Received: 27 Aug 2018, Revised: 28 Oct 2018, Accepted: 19 Dec 2018. 
labelling is said to be graceful. Not too many classes of trees are yet known to be graceful (see [1]). Erdös in an unpublished paper said that most graphs are not graceful, but graphs with some regularity are graceful (see [1]). Especially with trees, the conjecture of Alexander Rosa (see [1]) saying that all trees are graceful, has motivated many published papers regarding graceful trees. There are many classes of trees known to be graceful. Here we construct some classes of trees which are then proven to be graceful.

\section{Results}

In this section we will observe some classes of trees which then are known to be graceful.

\section{Uniform Caterpillars}

Denote by $P_{x}$ the path of $x$ vertices. One end vertex of a path will be called the head and the other one end will be called the back of the path. A uniform $(q, r)$-caterpillar or shortly $(q, r)$ caterpillar is a tree which is obtained by appending $r$ leaves to each vertex of $P_{q}$ except the back which is appended by $r+1$ leaves. The path $P_{q}$ will be called the backbone of $(q, r)$-caterpillar. It was proved in [2] that for any positive integers $q$ and $r,(q, r)$-caterpillar is graceful. Furthermore, it is clear that the number of vertices in a uniform $(q, r)$-caterpillar is equal to $q(r+1)+1$. A tree which is constructed from $p$ copies of $(q, r)$-caterpillars and a path $P_{p}$ by identifying the head of each $(q, r)$-caterpillar to exactly one vertex of $P_{p}$ is called a uniform super $(p, q, r)$-caterpillar, and is denoted by $S C(p, q, r)$. It is easy to check that the number of vertices of $S C(p, q, r)$ is equal to $p(1+q+q r)$.

The path $P_{p}$ is called the spine of $S C(p, q, r)$ (see [6]). For $r=0, S C(p, q, 0)$ is proved to be graceful in [6].

Without loss of generality, we count the vertices of the spine $P_{p}$ from one end point to the other one (from left to right) from 1 until $p$. Furthermore, we assign a number to each $(q, r)$-caterpillar corresponds to the number of vertex of $P_{p}$ on which the head of the caterpillar is identified. Denote each vertex of $S C(p, q, r)$ by $v_{i, j, k}$ with $i:=1,2, \ldots, r, r+1, j:=0,1,2, \ldots, q, k:=1,2, \ldots, p$, where $v_{r+1, j, k}$ are for the vertices in the backbone of the $k^{t h}(q, r)$-caterpillar which is at distant $j$ from the spine $P_{p}$, and $v_{i, j, k}$, with $j>0$, is the leaf of the $k^{t h}(q, r)$-caterpillar which is adjacent with vertex $v_{r+1, j-1, k}$.

Theorem 2.1. For every positive integers $p, q$, and $r$, the uniform super caterpillar $S C(p, q, r)$ is graceful.

Proof. Let $T(V, E):=C(p, q, r)$ and $s:=1+q+q r$. Here we have $|V|:=p s$ and $|E|:=p s-1$. Consider the following function $f$ from $V$ into $\{0,1,2, \ldots, p s-1\}$ and $f^{\prime}$ is a function from $E$ into $\{1,2, \ldots, p s-1\}$ such that $f^{\prime}(\{u, v\})=|f(u)-f(u)|$, for all $\{u, v\} \in E$.

For $i=1,2, \ldots, r, r+1, j=0,1,2, \ldots, q, k=1,2, \ldots, p$, we define the function $f$ as follows. 


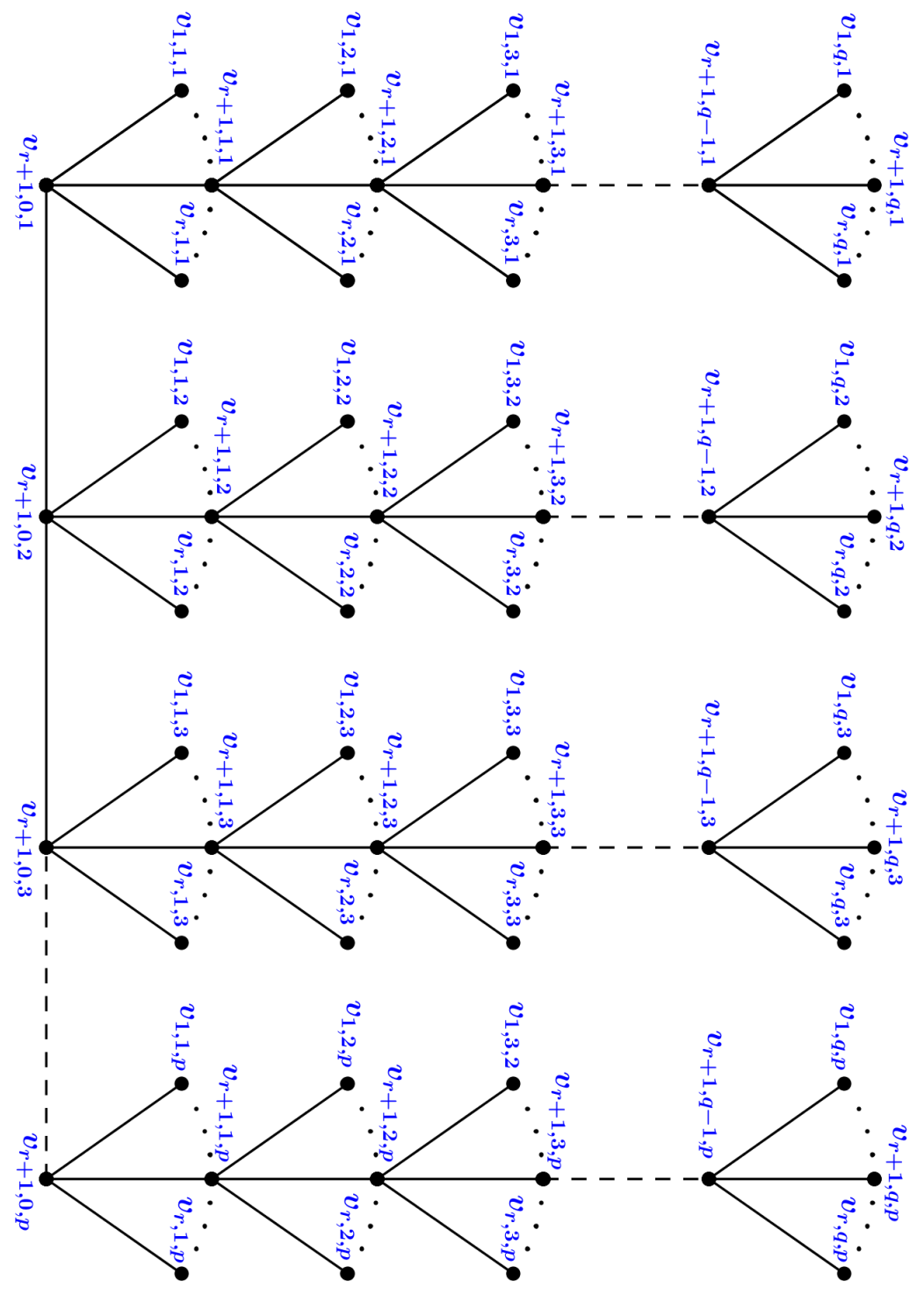

Figure 1. Graph of $S C(p, q, r)$

$$
f\left(v_{i, j, k}\right)= \begin{cases}\frac{1}{2}(k-1) s & \text { for } i=r+1, j=0, k \text { odd } \\ \frac{1}{2}(2 p-k) s & \text { for } i=r+1, j=0, k \text { even, } \\ i+\frac{1}{2}[(k-1) s+(j-2)(r+1)] & \text { for } j \neq 0 \text { even, } k \text { odd } \\ \frac{1}{2}[(2 p-(k-1)) s-(j-1)(r+1)]-i & \text { for } j \text { odd, } k \text { odd } \\ i+\frac{1}{2}[(2 p-k) s+(j-2)(r+1)] & \text { for } j \neq 0 \text { even, } k \text { even, } \\ \frac{1}{2}[k s-(j-1)(r+1)]-i & \text { for, } j \text { odd, } k \text { even. }\end{cases}
$$


This function induces the function $f^{\prime}$ as follows.

$$
\begin{aligned}
f^{\prime}\left(\left\{v_{r+1,0, k}, v_{r+1,0, k+1}\right\}\right)= & |(p-k) s|, \\
f^{\prime}\left(\left\{v_{i, j, k}, v_{r+1, j-1, k}\right\}\right)= & |(p-(k-1)) s-i-(j-1)(r+1)|, \\
& \text { for } j>0 \text { and } k \text { odd } \\
f^{\prime}\left(\left\{v_{i, j, k}, v_{r+1, j-1, k}\right\}\right)= & |(p-k) s+i+(j-1)(r+1)|, \\
& \text { for } j>0 \text { and } k \text { even }
\end{aligned}
$$

Now we want to show that $f$ is injective by showing that the range of $f$ is a subset of $\{0,1,2, \ldots, p s-$ $1\}$, and the cardinality of the range of $f$ is equal to $p s-1$.

Moreover, we will show that the range of $f^{\prime}$ is equal to the set of $\{1,2, \ldots, p s-1\}$, and therefore $f^{\prime}$ is bijective.

Let us denote the ranges of $f$ in Eq. (1) as $V_{1}^{\prime}, V_{2}^{\prime}, V_{3}^{\prime}$, and $V_{4}^{\prime}$, for the conditions of the pairs $(j, k)$ : (even, odd),(odd, odd),(even, even) and (odd, even), respectively. Thus for the whole range of $f$ is $V^{\prime}:=V_{1}^{\prime} \cup V_{2}^{\prime} \cup V_{3}^{\prime} \cup V_{4}^{\prime}$. Now we will describe $V_{i}^{\prime}, 1 \leq i \leq 4$. To this end, we will see each of the four cases of the pairs of $(p, q)$.

For the sake of space, we will present the proof only for the case $(p, q)=(\mathrm{even}$, even). The proof for other cases is similar.

For case $(p, q)=($ even, even) we have

$$
\begin{aligned}
V_{1}^{\prime}= & \left\{0, \ldots, \frac{s-1}{2}\right\} \cup\left\{s, \ldots, s+\frac{s-1}{2}\right\} \cup\left\{2 s, \ldots, 2 s+\frac{s-1}{2}\right\} \cup \ldots \\
& \cup\left\{\frac{(p-2) s}{2}, \ldots, \frac{(p-2) s}{s}+\frac{s-1}{2}\right\} \\
V_{2}^{\prime}= & \left\{\frac{(p+2) s}{2}-\frac{s-1}{2}, \ldots, \frac{(p+2) s}{2}-1\right\} \cup\left\{\frac{(p+4) s}{2}-\frac{s-1}{2}, \ldots, \frac{(p+4) s}{2}-1\right\} \cup \ldots \\
& \cup\left\{p s-\frac{s-1}{2}, \ldots, p s-1\right\} \\
V_{3}^{\prime}= & \left\{\frac{p s}{2}, \ldots, \frac{p s}{2}+\frac{s-1}{2}\right\} \cup\left\{\frac{(p+2) s}{2}, \ldots, \frac{(p+2) s}{2}+\frac{s-1}{2}\right\} \\
& \cup \ldots \cup\left\{(p-1) s, \ldots,(p-1) s+\frac{s-1}{2}\right\} \\
V_{4}^{\prime}= & \left\{s-\frac{s-1}{2}, \ldots, s-1\right\} \cup\left\{2 s-\frac{s-1}{2}, \ldots, 2 s-1\right\} \cup\left\{3 s-\frac{s-1}{2}, \ldots, 3 s-1\right\} \\
& \cup \ldots \cup\left\{\frac{p s}{2}-\frac{s-1}{2}, \ldots, \frac{p s}{2}-1\right\}
\end{aligned}
$$

Based on the above observation we get

$$
\begin{aligned}
V^{\prime}= & V_{1}^{\prime} \cup V_{2}^{\prime} \cup V_{3}^{\prime} \cup V_{4}^{\prime} \\
= & \left\{0, \ldots, \frac{s-1}{2}\right\} \cup\left\{s-\frac{s-1}{2}, \ldots, s-1\right\} \cup\left\{s, \ldots, s+\frac{s-1}{2}\right\} \\
& \cup\left\{2 s-\frac{s-1}{2}, \ldots, 2 s-1\right\} \cup \ldots \cup\left\{(p-1) s, \ldots,(p-1) s+\frac{s-1}{2}\right\} \\
& \cup\left\{p s-\frac{s-1}{2}, \ldots, p s-1\right\} \\
= & \{0,1,2, \ldots, p s-1\} .
\end{aligned}
$$

For this case we conclude that $f$ is an injection. By doing a similar way, we may conclude that $f$ is an injective function. 
Next we will show that the induced function $f^{\prime}$ is a bijective function. We will distinguish into two cases based on the kind of edges: $\left\{v_{r+1,0, k}, v_{r+1,0, k+1}\right\}$ and $\left\{v_{i, j, k}, v_{r+1, j-1, k}\right\}$.

i). For edges of the form $\left\{v_{r+1,0, k}, v_{r+1,0, k+1}\right\}, 1 \leq k<p$. Here we have $f^{\prime}\left(v_{r+1,0, k}, v_{r+1,0, k+1}\right)=$ $\left|f\left(v_{r+1,0, k}\right)-f\left(v_{r+1,0, k+1}\right)\right|$.

For $k$ odd we have

$$
\begin{aligned}
f^{\prime}\left(\left\{v_{r+1,0, k}, v_{r+1,0, k+1}\right\}\right) & =\left|\frac{1}{2}(k-1) s-\frac{1}{2}(2 p-(k+1)) s\right| \\
& =|k s-p s| \\
& =|(p-k) s| .
\end{aligned}
$$

For $k$ even we have

$$
\begin{aligned}
f^{\prime}\left(\left\{v_{r+1,0, k}, v_{r+1,0, k+1}\right\}\right) & =\left|\frac{1}{2}(2 p-k) s-\frac{1}{2}((k+1)-1) s\right| \\
& =|p s-k s| \\
& =|(p-k) s| .
\end{aligned}
$$

ii). For edges of the form $\left\{v_{i, j, k}, v_{r+1, j-1, k}\right\}$. We will divide into cases: $(k, j)=($ odd, odd $)$, $(k, j)=($ odd, even $),(k, j)=($ even, odd $)$, and $(k, j)=($ even, even $)$.

a) Case $(k, j)=($ odd, odd $)$.

Here we have

$$
\begin{aligned}
f^{\prime}\left(\left\{v_{i, j, k}, v_{r+1, j-1, k}\right\}\right)= & \mid\left(\frac{1}{2}((2 p-(k-1)) s-(j-1)(r+1))-i\right)- \\
& \left(r+1+\frac{1}{2}((k-1) s+((j-1)-2)(r+1)) \mid\right. \\
= & \mid\left(\frac{1}{2}((2 p-(k-1)) s-(j-1)(r+1))-i\right)- \\
& \frac{1}{2}((k-1) s+(j-1)(r+1)) \mid \\
= & \left|\frac{1}{2}(2 p-2(k-1)) s-i-\frac{1}{2}(2(j-1)(r+1))\right| \\
= & |(p-(k-1)) s-i-(j-1)(r+1)| .
\end{aligned}
$$

b) Case $(k, j)=($ odd, even $)$. In this case we have

$$
\begin{aligned}
f^{\prime}\left(\left\{v_{i, j, k}, v_{r+1, j-1, k}\right\}\right)= & \mid i+\frac{1}{2}((k-1) s+(j-2)(r+1))- \\
& \left(\frac{1}{2}((2 p-(k-1)) s-((j-1)-1)(r+1))-(r+1)\right) \mid \\
= & \mid i+\frac{1}{2}((k-1) s-(j-2)(r+1))- \\
& \frac{1}{2}((2 p-(k-1)) s-j(r+1)) \mid \\
= & \left|i+\frac{1}{2}(2(k-1)-2 p) s+\frac{1}{2}(2 j-2)(r+1)\right| \\
= & |(p-(k-1)) s-i-(j-1)(r+1)| .
\end{aligned}
$$

Thus, for Cases $(k, j)=($ odd, odd $)$ and $(k, j)=($ odd, even $)$ we have that $f^{\prime}\left(v_{i, j, k}, v_{r+1, j-1, k}\right)=$ $|(p-(k-1)) s-i-(j-1)(r+1)|$.

By doing a similar algebraic process we will get $f^{\prime}\left(v_{r+1,0, k}, v_{r+1,0, k+1}\right)=\mid(p-k) s+i+(j-$ $1)(r+1) \mid$ for the cases $(k, j)=($ even, odd $)$ and $(k, j)=($ even, even $)$.

Now let the image of $f^{\prime}\left(\left\{v_{r+1,0, k}, v_{r+1,0, k+1}\right\}\right)$ is $E_{1}^{\prime}$; of $f^{\prime}\left(\left\{v_{i, j, k}, v_{r+1, j-1, k}\right\}\right)$, for the cases $(k, j)=($ odd, odd $)$ and $(k, j)=($ odd, even $)$, is $E_{2}^{\prime}$; and the image of $f^{\prime}\left(\left\{v_{i, j, k}, v_{r+1, j-1, k}\right\}\right)$, for 
the cases $(k, j)=($ even, odd $)$ and $(k, j)=($ even, even $)$, is $E_{3}^{\prime}$. Furthermore, let $f^{\prime}(E)=E^{\prime}$. Thus $E^{\prime}=E_{1}^{\prime} \cup E_{2}^{\prime} \cup E_{3}^{\prime}$. We then have that $E_{1}^{\prime}=\{s, 2 s, \ldots,(p-1) s\}$. While for $E_{2}^{\prime}$ and $E_{3}^{\prime}$ we will see in two cases: $\mathrm{p}$ odd and $\mathrm{p}$ even.

For $p$ odd we have

$$
\begin{aligned}
E_{2}^{\prime}= & \{1,2, \ldots, s-1\} \cup\{2 s+1,2 s+2, \ldots, 3 s-1\} \cup \ldots \\
& \cup\{(p-1) s+1,(p-1) s+2, \ldots, p s-1\}, \text { and } \\
E_{3}^{\prime}= & \{s+1, s+2, \ldots, 2 s-1\} \cup\{3 s+1,3 s+2, \ldots, 4 s-1\} \cup \\
& \ldots \cup\{(p-2) s+1,(p-2) s+2, \ldots,(p-1) s-1\} .
\end{aligned}
$$

For case $p$ odd we get that $E^{\prime}=E_{1}^{\prime} \cup E_{2}^{\prime} \cup E_{3}^{\prime}=\{1,2, \ldots, p s-1\}$.

For $p$ even we have

$$
\begin{aligned}
E_{2}^{\prime}= & \{s+1, s+2, \ldots, 2 s-1\} \cup\{3 s+1,3 s+2, \ldots, 4 s-1\} \cup \\
& \ldots \cup\{(p-1) s+1,(p-1) s+2, \ldots,(p-1) s-1\}, \text { and } \\
E_{3}^{\prime}= & \{1,2, \ldots, s-1\} \cup\{2 s+1,2 s+2, \ldots, 3 s-1\} \cup \ldots \\
& \cup\{(p-2) s+1,(p-2) s+2, \ldots,(p-1) s-1\} .
\end{aligned}
$$

So, for case $p$ even, again we obtain that $E^{\prime}=E_{1}^{\prime} \cup E_{2}^{\prime} \cup E_{3}^{\prime}=\{1,2, \ldots, p s-1\}$. Thus, in all cases of $p$, we can conclude that $f^{\prime}(E)=E^{\prime}=\{1,2, \ldots, p s-1\}$. Since $|E|=\left|f^{\prime}(E)\right|=p s-1$, we may immediately infer that the function $f^{\prime}$ is bijective from $E$ onto $\{1,2, \ldots, p s-1\}$, and hence the function $f$ is a graceful labelling.

\section{Uniform Super Lobster Tree}

Here we will observe another class of trees which are graceful. These trees are called super uniform lobsters.

Consider a $(q, r)$-caterpillar. If we append $s$ leaves to each leaf of this $(q, r)$-caterpillar, except one leaf which is adjacent to the back, then we obtain a new tree which is called a uniform $(q, r, s)$ lobster. The leaf of the $(q, r)$-caterpillar which is not appended by $s$ leaves will be called the tail of the uniform $(q, r, s)$-lobster. The uniform $(q, r, s)$-lobster is called 2-distant tree in [2]. The number of vertices in a uniform $(q, r, s)$-lobster is equal to $q(r s+r+1)+1$. We will call again the path $P_{q}$ as the backbone of the uniform $(q, r, s)$-lobster.

For a positif integer $p>1$, take $p$ copies of uniform $(q, r, s)$-lobster and then identify their all tails. The resulting tree is called a uniform $(p, q, r, s)$-super lobster or shortly a $(p, q, r, s)$-super lobster, which is denoted by $S L(p, q, r, s)$. For $r=0,(p, q, r, 0)$-super lobster in [4] is called super caterpillar, and was proved to be graceful. Later we will show that $S L(p, q, r, s)$ is graceful.

First, we name the vertices of $S L(p, q, r, s)$ with $v_{i, j, k}$ and with $v_{i, j, k}^{h}$ where $i=1,2, \ldots, q$, $j=0,1,2, \ldots, r, k=1,2, \ldots, p, h=1,2, \ldots, s$, with the following details: 
$v_{i, 0, k}$ for the vertex which is on the $k^{t h}$ backbone and at distant $i$ from the center $v_{0,0,0}$; $v_{i, j, k}, j \neq 0$, for the vertex which is adjacent to $v_{i, 0, k}$; and $v_{i, j, k}^{h}$ for the leaf which is adjacent to $v_{i, j, k}$.

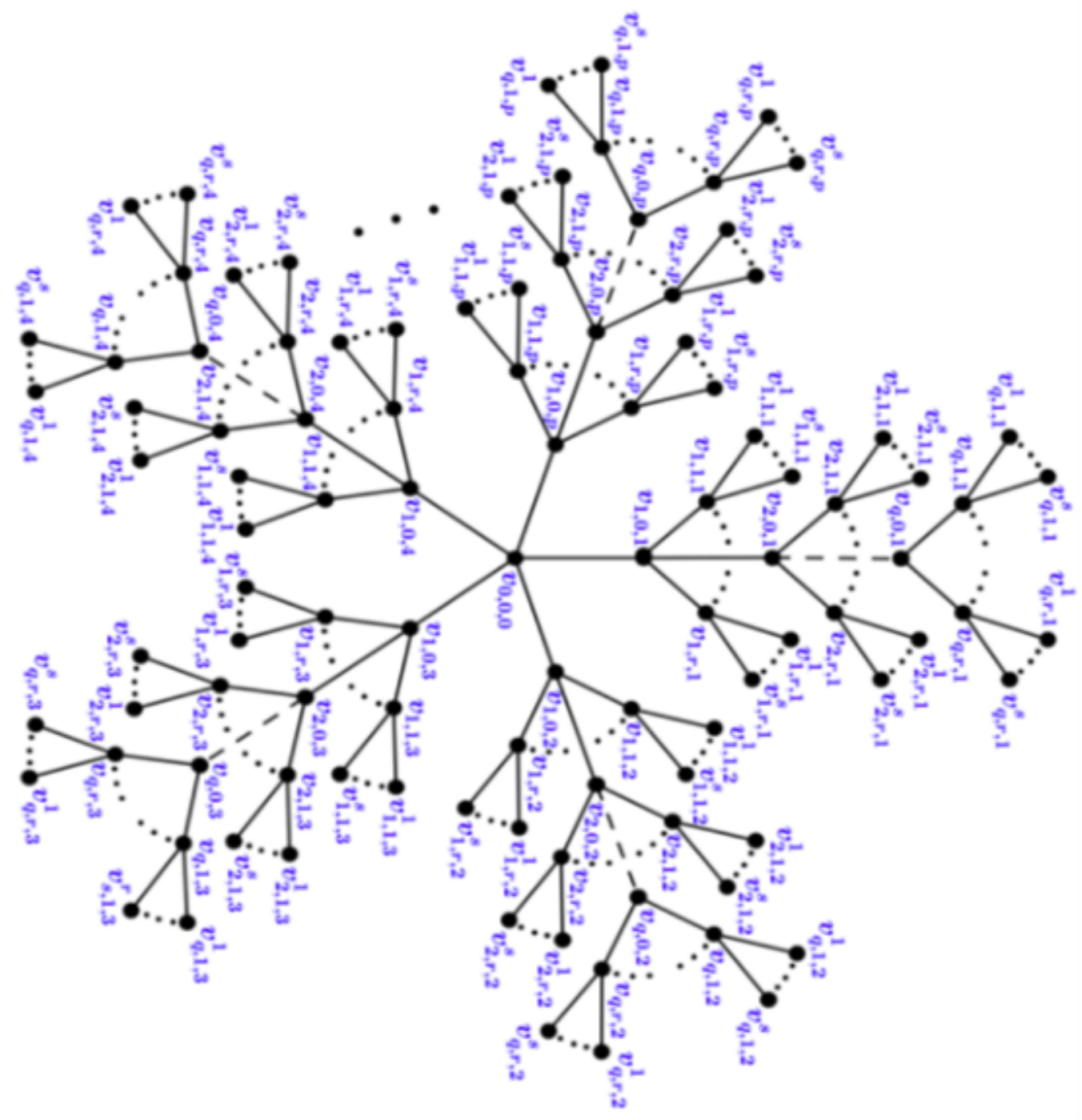

Figure 2. Diagram of $S L(p, q, r, s)$

Let $G(V, E):=S L(p, q, r, s)$ and $m=r(s+1)+1$. It is easy to see that $|V|=p q m+1$. Now we define a function $f$ from $V$ into $\{0,1,2, \ldots, p q m\}$ as the following.

For $1 \leq k \leq p$, we define 


$$
f\left(v_{i, j, k}\right)= \begin{cases}0 & \text { if } i=j=k=0, \\ p q m-\frac{(i-1) m}{2}-(k-1) q m, & \text { if } j=0 \text { and } i \text { odd } \\ \frac{i m}{2}+(k-1) q m, & \text { if } j=0 \text { and } i \text { even } \\ \frac{(i-1) m}{2}+(j-1)(s+1)+(k-1) q m+1, & \text { if } j>0 \text { and } i \text { odd } \\ p q m-\frac{(i-2) m}{2}-j(s+1)-(k-1) q m, & \text { if } j>0 \text { and } i \text { even }\end{cases}
$$

and for $1 \leq k \leq p$ and $0<j \leq r$ we define

$$
f\left(v_{i, j, k}^{h}\right)= \begin{cases}p q m-\frac{(i-1) m}{2}-(j-1)(s+1)-(k-1) q m-h, & \text { if } i \text { odd, } \\ \frac{(i-2) m}{2}+(j-1)(s+1)+(k-1) q m+h+1, & \text { if } i \text { even. }\end{cases}
$$

Theorem 2.2. For all positive integers $p, q, r$, and $s$, super lobster graph $S L(p, q, r, s)$ is graceful.

Proof. Consider the function defined in Eq. (7) and Eq. (8). Let $V_{1}^{\prime}, V_{2}^{\prime}, V_{3}^{\prime}, V_{4}^{\prime}, V_{5}^{\prime}, V_{6}^{\prime}, V_{7}^{\prime}$, stand for the ranges of $f$ for conditions $(i, j, k)=(0,0,0) ;(i$ odd, $j=0, k) ;(i$ even, $j=0, k) ;(i$ odd, $j>0, k)$; $(i$ even, $j>0, k)$; $(i$ odd, $j, k)$; and ( $i$ even, $j, k)$; respectively. Furthermore, let $V^{\prime}=V_{1}^{\prime} \cup V_{2}^{\prime} \cup V_{3}^{\prime} \cup V_{4}^{\prime} \cup V_{5}^{\prime} \cup V_{6}^{\prime} \cup V_{7}^{\prime}$.

First we will prove that $f$ is an injection.

We partition into two cases on $q$ : (i) $q$ is even, and (ii) $q$ is odd.

(i) Case $q$ is even. Here we have

$$
\begin{aligned}
A= & V_{3}^{\prime} \cup V_{4}^{\prime} \cup V_{7}^{\prime} \\
= & \left\{1,2, \ldots, \frac{q m}{2}\right\} \cup\left\{q m+1, \ldots, q m+\frac{q m}{2}\right\} \cup\left\{2 q m+1, \ldots, 2 q m+\frac{q m}{2}\right\} \cup \ldots \\
& \cup\left\{(p-1) q m+1, \ldots,(p-1) q m+\frac{q m}{2}\right\}, \text { and } \\
B= & V_{2}^{\prime} \cup V_{5}^{\prime} \cup V_{6}^{\prime} \\
= & \left\{\frac{q m}{2}+1, \ldots, q m\right\} \cup\left\{\frac{q m}{2}+q m+1, \ldots, 2 q m\right\} \cup\left\{\frac{q m}{2}+2 q m+1, \ldots, 3 q m\right\} \cup \ldots \\
& \cup\left\{(p-1) q m+\frac{q m}{2}+1, \ldots, p q m\right\} .
\end{aligned}
$$

By doing some algebraic calculations, we obtain that $V^{\prime}=V_{1}^{\prime} \cup A \cup B=\{0,1,2, \ldots, p q m\}$.

(ii) Case $q$ is odd.

Let $A=V_{3}^{\prime} \cup V_{4}^{\prime} \cup V_{7}^{\prime}$. In this case we will calculate $A$ into two phases with respect to $q$ : for $0<i<q$, and then for $i=q$.

Let $A_{1}=V_{3}^{\prime} \cup V_{4}^{\prime} \cup V_{7}^{\prime}$ for $0<i<q$, and $A_{2}=V_{3}^{\prime} \cup V_{4}^{\prime} \cup V_{7}^{\prime}$ for $i=q$. Thus the total $V_{3}^{\prime} \cup V_{4}^{\prime} \cup V_{7}^{\prime}=A_{1} \cup A_{2}$. Here we have that

$$
\begin{aligned}
A_{1}= & \left\{1, \ldots, \frac{(q-1) m}{2}\right\} \cup\left\{q m+1, \ldots, q m+\frac{(q-1) m}{2}\right\} \cup\left\{2 q m+1, \ldots, 2 q m+\frac{(q-1) m}{2}\right\} \\
& \cup \ldots \cup\left\{(p-1) q m+1, \ldots,(p-1) q m+\frac{(q-1) m}{2}\right\}, \text { and } \\
A_{2}= & \left\{\frac{(q-1) m}{2}+1, \frac{(q-1) m}{2}+(s+1)+1, \ldots, \frac{(q+1) m}{2}-(s+1)\right\} \\
& \cup\left\{q m+\frac{(q-1) m}{2}+1, q m+\frac{(q-1) m}{2}+(s+1)+1, \ldots, q m+\frac{(q+1) m}{2}-(s+1)\right\} \\
& \cup \ldots \cup\left\{(p-1) q m+\frac{(q-1) m}{2}+1,(p-1) q m+\frac{(q-1) m}{2}+(s+1)+1, \ldots,\right. \\
& \left.(p-1) q m+\frac{(q+1) m}{2}-(s+1)\right\} .
\end{aligned}
$$


Furthermore, let $B=V_{2}^{\prime} \cup V_{5}^{\prime} \cup V_{6}^{\prime}$. As we did above, here again we will calculate $B$ into two phases with respect to $q$ : for $0<i<q$, and then for $i=q$.

Let $B_{1}=V_{2}^{\prime} \cup V_{5}^{\prime} \cup V_{6}^{\prime}$ for $0<i<q$, and $B_{2}=V_{2}^{\prime} \cup V_{5}^{\prime} \cup V_{6}^{\prime}$ for $i=q$. Thus the total $V_{2}^{\prime} \cup V_{5}^{\prime} \cup V_{6}^{\prime}=B_{1} \cup B_{2}$. We obtain the following result.

$$
\begin{aligned}
B_{1}= & \left\{\frac{(q+1) m}{2}+1, \ldots, q m\right\} \cup\left\{q m+\frac{(q+1) m}{2}+1, \ldots, 2 q m\right\} \cup \\
& \left\{2 q m+\frac{(q+1) m}{2}+1, \ldots, 3 q m\right\} \cup \ldots \cup \\
& \left\{(p-1) q m+\frac{(q+1) m}{2}+1, \ldots, p q m\right\}, \text { and } \\
B_{2}= & \bigcup_{k=1}^{p}\left(\left\{(k-1) q m+\frac{(q-1) m}{2}+2, \ldots,(k-1) q m+\frac{(q-1) m}{2}+(s+1)\right\}\right. \\
& \cup\left\{(k-1) q m+\frac{(q-1) m}{2}+(s+1)+2, \ldots,(k-1) q m+\frac{(q-1) m}{2}+2(s+1)\right\} \\
& \cup \ldots \cup\left\{(k-1) q m+\frac{(q+1) m}{2}-(s+1)+1, \ldots,(k-1) q m+\frac{(q+1) m}{2}-1\right\} \\
& \left.\left.\cup(k-1) q m+\frac{(q+1) m}{2}\right\}\right) .
\end{aligned}
$$

Thus we have

$$
\begin{aligned}
V^{\prime}= & V_{1}^{\prime} \cup A_{1} \cup A_{2} \cup B_{1} \cup B_{2} \\
& \{0\} \cup\left\{1, \ldots, \frac{(q-1) m}{2}, \frac{(q-1) m}{2}+1, \ldots, \frac{(q+1) m}{2}, \frac{(q+1) m}{2}+1, \ldots, q m\right\} \\
& \cup\left\{q m+1, \ldots, q m+\frac{(q-1) m}{2}, q m+\frac{(q-1) m}{2}+1, \ldots, q m+\frac{(q+1) m}{2},\right. \\
& \left.q m+\frac{(q+1) m}{2}+1, \ldots, 2 q m\right\} \cup \ldots \cup\left\{(p-1) q m+1, \ldots,(p-1) q m+\frac{(q-1) m}{2},\right. \\
& \left.(p-1) q m+\frac{(q-1) m}{2}+1, \ldots,(p-1) q m+\frac{(q+1) m}{2},(p-1) q m+\frac{(q+1) m}{2}+1, \ldots, p q m\right\} \\
= & \{0,1,2, \ldots, p q m\} .
\end{aligned}
$$

We see that for every case we obtain that $V_{1}^{\prime} \cup V_{2}^{\prime} \cup V_{3}^{\prime} \cup V_{4}^{\prime} \cup V_{5}^{\prime} \cup V_{6}^{\prime} \cup V_{7}^{\prime}=\{0,1,2, \ldots, p q m\}$. Thus the function $f$ is not only injective but also bijective. We will leave the proof that the function $f^{\prime}$ is bijective from $E$ onto $\{1,2, \ldots, p q m\}$.

\section{Uniform Distant Trees}

The third class of trees we want to show to be graceful is the class of uniform distant trees. A tree in which all internal vertices have degrees $r+1$ except one internal vertex, is called an full $r$-ary tree. the vertex of degrees $r$ will be called the root of the tree. A uniform full $r$-ary tree is a full r-ary tree in which all of its leaves are at the same level, say $q$, and is denoted by $T_{q}^{r}$. A tree which is obtained from $p$ copies of $T_{q}^{r}$ and a path of $p$ vertices, $P_{p}$, by identifying each vertex of $P_{p}$ with exactly the root of each $T_{q}^{r}$ is called a uniform $(p, q, r)$-distant tree and will be denoted by $D(p, q, r)$. In [6] it was proved that $D(p, q, 1)$ is graceful. Here we extend this result for $r>1$.

It is easy to see that the order of $T_{q}^{r}$ is $\frac{r^{q+1}-1}{r-1}$, and hence the order of $D(p, q, r)$ is $p s$ with $s=$ $\frac{r^{q+1}-1}{r-1}$.

Now we want to name vertices of $D(p, q, r)$ to facilitate the definition of a function from the set of vertices to the set $\{0,1,2, \ldots, p s-1\}$. First we name $p$ vertices of the path $P_{p}$ from one end to the other by 1 up until $p$. If the root of the tree $T_{q}^{r}$ is identified with the $k^{\text {th }}$ vertex of the path $P_{p}$., then we call the tree as the $k^{t h}$ tree in $P_{p}$. We name vertices as $v_{i_{j}, j, k}$ which has the following details: 
i) $j=0,1, \ldots, q$ for referring the distance of the vertex from the path $P_{p}$.

ii) $i=1,2, \ldots, r^{j}$ for referring the order of vertices at level $j$ in each $T_{q}^{r}$.

iii) $k=1,2, \ldots, p$ indicates that the vertex is in the $k^{\text {th }}$ tree $T_{q}^{r}$.

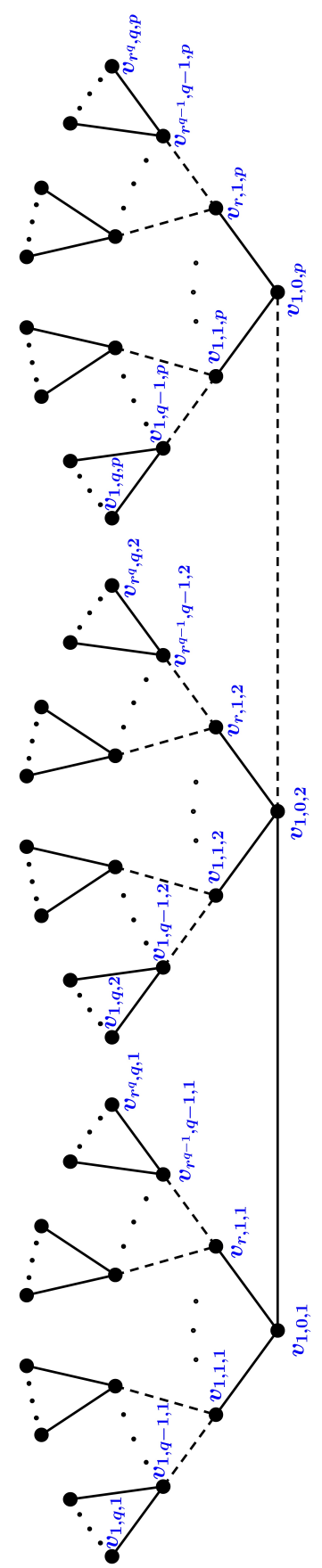

Figure 3. Diagram of $D(p, q, r)$

For labelling vertices in $D(p, q, r)$ we define a function $f_{n}$ from $\{0,1,2, \ldots, p s-1\}$ with 
$n=1,2, \ldots, q$ as follows.

$$
f_{1}\left(v_{1,0,1}\right)=0 \text {, then let } n=1,2, \ldots, q .
$$

For $k=1$ we will see two cases: $1 \leq j<n$ and $j=n$.

For case $1 \leq j<n$, we define

$$
f_{n}\left(v_{i, j, k}\right)= \begin{cases}f_{n-1}\left(v_{i, j, k}\right)+r^{n} \frac{(i-1)}{r^{j}}, & \text { if } j \text { even and } i=1,2, \ldots, r^{j} \\ f_{n-1}\left(v_{i, j, k}\right)+r^{n}\left(p-\frac{i-1}{r^{j}}\right), & \text { if } j \text { odd and } i=1,2, \ldots, r^{j}\end{cases}
$$

For $j=n$ let $m=1,2, \ldots, r^{n-1}$ and $i=1+r(m-1), \ldots, r+r(m-1)$. Here we define

$$
f_{n}\left(v_{i, n, k}\right)=f_{n}\left(v_{r^{n-1}-m+1, n-1, k}\right)+(-1)^{n-1}\left((r m-i+1)+(p-1) s_{n}\right),
$$

with $s_{n}=\frac{r^{n+1}-1}{r-1}$.

For $1<k \leq p, 0 \leq j \leq q$, and $1 \leq i \leq r^{j}$, we define

$$
f_{n}\left(v_{i, j, k}\right)= \begin{cases}f_{n}\left(v_{i, j, 1}\right)+(-1)^{j}\left(\frac{k-1}{2}\right) s_{n}, & \text { if } k \text { odd } \\ f_{n}\left(v_{i, j, 1}\right)+(-1)^{j}\left(p-\frac{k}{2}\right) s_{n}, & \text { if } k \text { even }\end{cases}
$$

Theorem 2.3. For all positive integers $p, q$, and $r \geq 2$, the graph $D(p, q, r)$ is graceful.

Proof. Let $G(V, E)$ be the graph $D(p, q, r)$. Consider the function $f$ as defined in Eqs. 9, 10, and 11 above. We define a function $f^{\prime}$ from $E$ onto $\{1,2, \ldots, p s-1\}$ such that for every edge $u v \in E$, $f^{\prime}(u v)=|f(u)-f(v)|$. Next we will show that $f$ and $f^{\prime}$ are injective and bijective, respectively. First we want to show that the function $f$ is injective. Let the ranges of $f$ eqs. 9, 10, and 11 be $V_{1}^{\prime}, V_{2}^{\prime}$, and $V_{3}^{\prime}$ respectively. Thus the total range of $f$ is $V^{\prime}:=V_{1}^{\prime} \cup V_{2}^{\prime} \cup V_{3}^{\prime}$. Consider now the cases of $p$.

First, assume that $p$ is even. For the case $k$ odd, $j$ even, or the case $k$ even, $j$ odd, let $A:=$ $V_{1}^{\prime} \cup V_{2}^{\prime} \cup V_{3}^{\prime}$. Then we have that

$$
\begin{aligned}
A= & \{0,1, \ldots, s-1\} \cup\{s, s+1, \ldots, 2 s-1\} \cup\{2 s, 2 s+1, \ldots, 3 s-1\} \\
& \cup\{3 s, 3 s+1, \ldots, 4 s-1\} \cup \ldots \cup\left\{\left(\frac{p}{2}-1\right) s,\left(\frac{p}{2}-1\right) s+1, \ldots, \frac{p s}{2}-1\right\} .
\end{aligned}
$$

Moreover, for the case $k$ odd, $j$ odd, or the case $k$ even, $j$ even, let $B:=V_{1}^{\prime} \cup V_{2}^{\prime} \cup V_{3}^{\prime}$. Then we get

$$
\begin{aligned}
B= & \left\{\frac{p s}{2}, \frac{p s}{2}+1, \ldots,\left(\frac{p}{2}+1\right) s-1\right\} \cup\left\{\left(\frac{p}{2}+1\right) s,\left(\frac{p}{2}+1\right) s+1, \ldots,\left(\frac{p}{2}+2\right) s-1\right\} \cup \\
& \left\{\left(\frac{p}{2}+2\right) s,\left(\frac{p}{2}+2\right) s+1, \ldots,\left(\frac{p}{2}+3\right) s-1\right\} \cup \ldots \cup \\
& \{(p-1) s,(p-1) s+1, \ldots, p s-1\} .
\end{aligned}
$$

Thus $V^{\prime}=A \cup B=\{0,1,2, \ldots, p s-1\}$.

Secondly, consider the case $p$ odd. Here, we will partition into case $1 \leq k<p$ and case $k=p$. Let $V_{1}^{\prime} \cup V_{2}^{\prime} \cup V_{3}^{\prime}=A \cup B \cup C$.

For the case $k$ odd, $j$ even, or the case $k$ even, $j$ odd, let $A:=V_{1}^{\prime} \cup V_{2}^{\prime} \cup V_{3}^{\prime}$. Then we have that 


$$
\begin{aligned}
A= & \{0,1, \ldots, s-1\} \cup\{s, s+1, \ldots, 2 s-1\} \cup\{2 s, 2 s+1, \ldots, 3 s-1\} \\
& \cup\{3 s, 3 s+1, \ldots, 4 s-1\} \cup \ldots \cup\left\{\left(\frac{p-3}{2}\right) s,\left(\frac{p-3}{2}\right) s+1, \ldots,\left(\frac{p-1}{2}\right) s-1\right\} .
\end{aligned}
$$

Then, for the case $k$ odd, $j$ odd, or the case $k$ even, $j$ even, let $B:=V_{1}^{\prime} \cup V_{2}^{\prime} \cup V_{3}^{\prime}$. Then we get

$$
\begin{aligned}
B= & \left\{\frac{(p+1) s}{2}, \frac{(p+1) s}{2}+1, \ldots, \frac{(p+3) s}{2}-1\right\} \cup\left\{\frac{(p+3) s}{2}, \frac{(p+3) s}{2}+1, \ldots, \frac{(p+5) s}{2}-1\right\} \cup \\
& \left\{\frac{(p+5) s}{2}, \frac{(p+5) s}{2}+1, \ldots, \frac{(p+7) s}{2}-1\right\} \cup \ldots \cup\{(p-1) s,(p-1) s+1, \ldots, p s-1\} .
\end{aligned}
$$

For $k=p$, we have $C:=V_{1}^{\prime} \cup V_{2}^{\prime} \cup V_{3}^{\prime}=\left\{\left(\frac{p-1}{2}\right) s,\left(\frac{p-1}{2}\right) s+1, \ldots\left(\frac{p+1}{2}\right) s-1\right\}$. Thus, by some simple algebraic calculations we obtain $V^{\prime}=A \cup B \cup C=\{0,1,2, \ldots, p s-1\}$.

Since for every case we have that the total image of $f$ is $V^{\prime}=\{0,1,2, \ldots, p s-1\}$, and hence $\left|V^{\prime}\right|=|V|$, then we may conclude that $f$ is an injection (even a bijection).

Next, it remains to show that the induced function $f^{\prime}$ is a bijection.

a. First let us consider edges $\left\{v_{1,0, k}, v_{1,0, k+1}\right\}$. For $n=1,2, \ldots, q$ let $s_{n}=\frac{r^{n+1}-1}{r-1}$. Here we have $f_{n}^{\prime}\left(\left\{v_{1,0, k}, v_{1,0, k+1}\right\}\right)=\left|f_{n}\left(v_{1,0, k}\right)-f_{n}\left(v_{1,0, k+1}\right)\right|$. If $k$ is odd we obtain

$$
\begin{aligned}
f_{n}^{\prime}\left(\left\{v_{1,0, k}, v_{1,0, k+1}\right\}\right) & =\left|\left(f_{n}\left(v_{1,0,1}\right)+(-1)^{0}\left(\frac{k-1}{2}\right) s_{n}\right)-\left(f_{n}\left(v_{1,0,1}\right)+(-1)^{0}\left(p-\frac{k+1}{2}\right) s_{n}\right)\right| \\
& =\left|\left(\frac{k-1}{2}\right) s_{n}-\left(p-\frac{k+1}{2}\right) s_{n}\right| \\
& =\left|(p-k) s_{n}\right| .
\end{aligned}
$$

If $k$ is even, we obtain

$$
\begin{aligned}
f_{n}^{\prime}\left(\left\{v_{1,0, k}, v_{1,0, k+1}\right\}\right) & =\left|\left(f_{n}\left(v_{1,0,1}\right)+(-1)^{0}\left(p-\frac{k}{2}\right) s_{n}\right)-\left(f_{n}\left(v_{1,0,1}\right)+(-1)^{0}\left(\frac{(k+1)-1}{2}\right) s_{n}\right)\right| \\
& =\left|\left(p-\frac{k}{2}\right) s_{n}-\left(\frac{k}{2}\right) s_{n}\right| \\
& =\left|(p-k) s_{n}\right| .
\end{aligned}
$$

From here we have

$$
f_{n}^{\prime}\left(\left\{v_{1,0, k}, v_{1,0, k+1}\right\}\right)=\left|(p-k) s_{n}\right| .
$$

b. Now we consider the edges $\left\{v_{i, j, k}, v_{t, j+1, k}\right\}$ with $i=1,2, \ldots, r$ and $t=i r-(r-1), i r-(r-2), \ldots, i r$. Here we have

$$
f_{n}^{\prime}\left(\left\{v_{i, j, k}, v_{t, j+1, k}\right\}\right)=\left|f_{n}\left(v_{i, j, k}\right)-f_{n}\left(v_{t, j+1, k}\right)\right| .
$$

Let the images of $f_{n}^{\prime}$ in Eqs.(12) and (13) are $E_{1}^{\prime}$ and $E_{2}^{\prime}$ respectively, and the total image of $f_{n}^{\prime}$ is $E^{\prime}$. Then, $E^{\prime}=E_{1}^{\prime} \cup E_{2}^{\prime}$. Furthermore, we have that

$$
\begin{aligned}
E_{1}^{\prime}= & \{(p-1) s,(p-2) s,(p-3) s, \ldots, s\}, \text { and } \\
E_{2}^{\prime}= & \{p s-1, \ldots,(p-1) s+1\} \cup\{(p-1) s-1, \ldots,(p-2) s+1\} \cup \\
& \{(p-2) s-1, \ldots,(p-3) s+1\} \cup \ldots \cup\{s-1, \ldots, 1\} .
\end{aligned}
$$

Then $E^{\prime}=E_{1}^{\prime} \cup E_{2}^{\prime}=\{1,2, \ldots, p s-1\}$. Thus, we can see that in any case we have that $|E|=p s-1=\left|E^{\prime}\right|$. This means that the function $f^{\prime}$ is a bijection from $E$ onto $E^{\prime}=\{1,2, \ldots, p s-1\}$. We are done.

\section{Conclusion}

We should mention here that all uniform trees in this paper can indeed be proven by the method introduced in [3], but in this paper we label the whole graph directly, whereas in [3] the labeling proses is carried out part by part. 


\section{Acknowledgement}

The authors thank to anonimous refrees for their useful notions.

\section{References}

[1] J.A. Gallian, A Dynamic Survey of Graph Labelling, Elect. J. Combinatorics, (2017).

[2] D. Morgan, All Lobsers with Perfect Matchings are Graceful, Electronic Notes in Discrete Mathematics, Vol 11 (2002), 503-508.

[3] K.M. Koh, D.G. Rogers and T. Tan, Products of graceful trees, Discrete Mathematics, Vol. 31 (1980), 279-292.

[4] A. Munia, J. Maowa, S. Tania, M. Kaykobad,A New Class of Graceful Tree. International Journal of Engineering Sciences and Research, Vol 5 (2014), 1112-1115.

[5] N. Ujwala, Applications of Graceful Graph, International Journal of Engineering Sciences and Research Technology, Vol 4 (2015), pp.129-131.

[6] K. Wenger, Two Rosa-type Labeling of Uniform $k$-distant Trees and a New Class of Trees, Honors Projects in Mathematics of Illnois Wesleyan University, (2015). 